\title{
Descolonizar la economía: espacios de economías diversas y ontologías mapuche en Alto Biobío, Chile ${ }^{1}$
}

\author{
Marcela Palomino-Schalscha ${ }^{2}$
}

\begin{abstract}
RESUMEN
Las economías mapuche han sido comúnmente descritas como cerradas y de subsistencia, invisibilizando cómo contribuyen y diversifican la economía. Examinando el caso de Trekaleyin, una iniciativa turística mapuche-pewenche en Alto Biobío, este artículo explora desde perspectivas diversas y decoloniales las configuraciones económicas en las que está articulada. Basado en trabajo de campo usando metodologías etnográficas y de investigación-acción, este artículo entrelaza debates actuales dentro de la geografía en torno a las economías diversas y el postdesarrollo con aspectos de las ontologías mapuche. Los resultados presentados corresponden al reconocimiento y análisis detallado de las múltiples prácticas, actores y ontologías en juego en estos ensamblajes económicos que van más allá del capitalismo. Se concluye que considerar estos elementos diversos usualmente excluidos de lo económico es crucial para reconocer el carácter performativo, híbrido y negociado de los ensamblajes económicos, y para avanzar hacia ideas y prácticas descolonizadoras acerca de la economía y el desarrollo.
\end{abstract}

Palabras clave: Economía diversa y comunitaria, descolonización, turismo indígena, mapuche, postdesarrollo.

\begin{abstract}
Mapuche economies have been commonly described as closed and focused only on subsistence, making the ways in which they contribute to and diversify the economy invisible. Examining the case of Trekaleyin, a Mapuche-Pewenche tourism initiative in Alto Biobío, this paper explores the economic context in which it was developed from a diverse and de-colonial perspectives. Based on fieldwork using ethnographic and Participatory Action-Research methodologies, this article brings together debates within geography on diverse economies and post-development and elements of Mapuche ontologies. The results presented, then, are based on the recognition and detailed analysis of multiple practices, actors and ontologies at play within these economic arrangements, which reach beyond capitalism. The paper concludes by considering these diverse elements, which are usually excluded from the economy, are key in recognizing the performative, hybrid and negotiated nature of economic arrangements, and in moving towards a decolonization of ideas and practices on the economy and development.
\end{abstract}

Key words: Diverse and community economies, decolonization, Indigenous tourism, Mapuche, post-development.

\footnotetext{
1 Artículo recibido el 30 de abril de 2015, aceptado el 15 de julio de 2015 y corregido el 3 de septiembre de 2015 .
}

\footnotetext{
2 School of Geography, Environment and Earth Sciences, Victoria University of Wellington (New Zealand). E-mail: marcela.palomino-schalscha@vuw.ac.nz
} 
El turismo indígena tiende a enfatizar la diferencia y otredad de los pueblos indígenas, transformándolos en algo exótico y atractivo (Mowforth et al., 2008). En Chile, los gobiernos de postdictadura han apoyado el desarroIlo del turismo indígena desde la década de los noventa a través de distintos programas y proyectos (Bushel y Salazar, 2009; Morales, 2006; Pilquimán y Skewes, 2009). El potencial turístico y comercial de la diferencia indígena, entendida como una "ventaja comparativa", ha sido una de las maneras en las que, a través de un enfoque multicultural neoliberal, estos gobiernos han "autorizado" e incluido a los mapuche, el pueblo indígena más numeroso en Chile, en políticas económicas y de desarrollo. Sin embargo, en términos generales este enfoque ha tendido a pasar por alto una serie de cuestiones que van desde lo material a lo ontológico, asumiendo que el desarrollo económico, incluyendo el turismo, se trata solo de incorporar y alinear a los pueblos indígenas con un orden económico supuestamente tan obvio e inevitable que ni siquiera es posible cuestionarlo (PalominoSchalscha, 2012).

Sin embargo, al examinar en detalle el turismo indígena resulta evidente que vincular turismo y desarrollo económico está lejos se ser tan simple, ya que involucra múltiples dinámicas y elementos, y entreteje diversos valores, saberes, prácticas y ontologías. Pero el hecho de que la economía en sí misma, y no solo entre de los pueblos indígenas está entrecruzada por elementos culturales y procesos socioeconómicos más amplios tales como significados, identidades y diversos saberes, ha sido un tema de considerable investigación desde el "giro cultural" en la geografía (Faulconbridge \& Hall, 2009). Distintos autores han cuestionado la idea de que la economía es una esfera compartimentalizada que funciona de acuerdo a reglas objetivas y sistemáticas, y han demostrado que está intrínsecamente conformada por elementos que escapan a lo que convencionalmente se considera como "lo económico", y que suelen ser borrados de discursos económicos (Amin \& Thrift, 2004). Dentro de esta línea teórica, Amin y Thrift (2007) han destacado la gran variedad de actores involucrados en la constitución de la economía, entendiéndola como el resultado de la mezcla de elementos híbridos con distintos objetivos, que incluyen entre otros dinero, saberes, objetos, tecnologías, normas, edificios, poder, pasiones y gente. Para ellos, entonces, la economía es el resultado de entretejer "reglas abstractas, legados históricos, prácticas materiales, narrativas simbólicas y discursivas, hábitos sociales y culturales, disposiciones materiales, emociones y aspiraciones" (Amin \& Thrift, 2007: 145, traducción propia).

En gran medida debido a interpretaciones limitadas de lo que constituye la economía, los sistemas económicos mapuche han sido generalmente definidos como cerrados y solo enfocados en la subsistencia. Esto no solo pasa por alto una realidad histórica al invisibilizar su conectividad y contribución a la economía más amplia, por ejemplo desde los tiempos de la colonización española cuando sus redes de comercio se extendían desde Perú por el norte hasta Argentina por el este (COTAM, 2003b; Marimán et al., 2006; Pinto, 2003). El representar la economía mapuche desde una visión reductiva de lo económico, lleva a invisibilizar también un sinnúmero de relaciones, valores, marcos ontológicos y prácticas que la constituyen, lo que promueve concepciones eurocéntricas de la economía y el desarrollo que son presentados como la única opción posible y, por ende, la única vía para superar la pobreza. Esta mirada está lejos de ser inocente, ya que está fundada en una visión colonial que impone paradigmas de la euromodernidad que privilegian ciertos saberes y prácticas (modernos occidentales, incluyendo la economía como meramente capitalista), subordinando y descartando otras formas de saber y existir (Mignolo, 2009; Quijano, 2007).

En respuesta, este artículo aborda el estudio de la economía desde una perspectiva más amplia y descolonizadora. Me enfocaré en Trekaleyin, una iniciativa turística mapuche-pewenche relativamente pequeña que opera desde el año 2004, recibiendo principalmente turistas chilenos pero también ocasionalmente extranjeros. Ubicada en la comuna de Alto Biobío (Figura $\mathrm{N}^{0} 1$ ), en la cordillera de los Andes cerca del límite con Argentina, Trekaleyin agrupa a cuatro de las cinco comunidades del Valle del Queuco, quienes ofrecen principalmente cabalgatas por sus sendas cordilleranas (Figura $N^{\circ} 2$ ). En particular, este artículo emerge de parte de mi investigación de doctorado (Palomino- 
Figura $\mathrm{N}^{\circ} 1$

Localización de la comuna de Alto Biobío
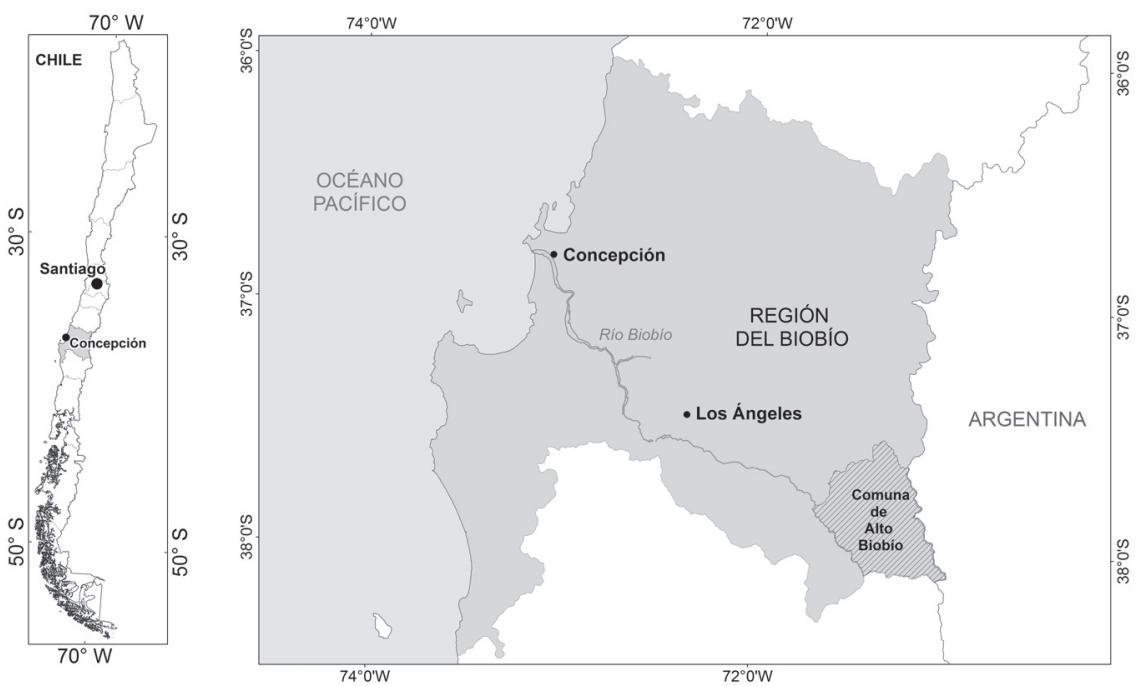

Fuente: Elaborado por Viviana Huiliñir-Curío.

Schalscha, 2012), la que involucró seis meses de terreno (noviembre 2008 a mayo 2009), y visitas en los años posteriores, trabajando desde una perspectiva etnográfica y de investigación-acción que incluyó la conducción de 21 entrevistas semiestructuradas y observación participante.

Analizando el caso de Trekaleyin, este artículo busca responder las siguientes interrogantes: ¿cuáles son los saberes, prácticas y valores que conforman las disposiciones económicas actuales de estas comunidades?, ¿Cómo se articula el turismo en ellas?, ¿cuáles son las dimensiones ontológicas de estas economías?, ¿cuáles son las consecuencias de entender la economía desde perspectivas diversas y descolonizantes? Para ello, me basaré en debates en la literatura en torno a la diferencia entre la economía de mercado y la comunitaria, para luego profundizar en el innovador trabajo de Gibson-Graham (2006) en torno a economías diversas desde perspectivas postcapitalistas y de postdesarrollo. Revisaré también perspectivas decoloniales con énfasis en lo económico, y acercamientos a las ontologías y enfoques económicos mapuche. Con ello, mi objetivo es demostrar los vínculos intrínsecos que la economía tiene con otras múltiples dimensiones y elementos, reconociendo prácticas, saberes, ontologías y actores generalmente invisibles en la mayoría de los discursos económicos. También, al entender la economía como perforada cotidianamente en formas materiales y simbólicas, busco desafiar ideas universales y simplistas de lo económico que la presentan como un ente monolítico y uniforme para abrir espacios de pluralidad.

Este artículo, entonces, responde a llamados desde las geografías postcoloniales a estudiar la configuración de economías indígenas y/o no occidentales, así como también a analizar en casos concretos la constitución de economías diversas (Amin \& Thrift, 2004; Gibson-Graham, 2006; Pollard et al., 2011; Zein-Elabdin \& Charusheela, 2004). Simultáneamente, al tomar en serio las ontologías mapuche como elementos relevantes en configuraciones económicas, contribuye a la descolonización de la economía tal como ha sido sugerido por Marimán et al. (2006). Por ello, desde una crítica decolonial, se busca cuestionar lo que Santos (2003) ha denominado el "desperdicio" de experiencias y saberes diversos que son desechados por la imposición de la modernidad occidental. 
Se busca así aportar a la descolonización de la economía, entendiendo el proceso de descolonización de acuerdo a lo propuesto por Grosfoguel (2007: 219) como un proceso que involucra la transformación de múltiples dimensiones que incluyen "las jerarquías sexuales, de género, espirituales, económicas, políticas, lingüísticas y raciales del sistemamundo colonial/moderno" como resultado de un diálogo entre diversos "proyectos epistémicos/éticos/políticos que lleven a un mundo pluriversal en vez uno de universal" (p. 212, traducción propia). Así, este artículo busca abrir espacios para pensar alternativas "impensables" al sistema económico y cultural occidental dominante (Blaser \& De la Cadena, 2009), y desafiar nociones dualistas y estáticas de lo tradicional y su supuesta separación de lo moderno (Escobar, 2005).

El presente artículo comienza, entonces, examinando debates en torno a la distinción entre economías comunitarias y de mercado. Luego introduce en detalle debates en la literatura en torno a las economías diversas y comunitarias para después explorar bajo este lente las configuraciones económicas de las comunidades involucradas en Trekaleyin, en particular atendiendo a las formas de desarrollar transacciones, remunerar el trabajo y estructurar las formas de producción. Posteriormente, se presenta una aproximación a la economía desde las ontologías mapuche, y cómo estas están en juego en la manera en que Trekaleyin opera y coconstituyen los ensamblajes económicos de estas comunidades. Finalmente este artículo cierra con las conclusiones generales de este trabajo y sus implicancias para la descolonización y el postdesarrollo.

\section{Aproximaciones a las economías comunitarias}

El trabajo de Tönnies, remontándose al año 1887 , ha modelado profundamente las ideas dualistas acerca de las comunidades y sus vínculos con la economía hasta hoy (Aitken, 2009). Tönnies distinguió lo que él llamó comunidades premodernas (Gemeinschaft) de las sociedades modernas (Gesellschaft). Según él, las comunidades están basadas en relaciones sociales cercanas donde el lugar y las relaciones cara a cara son claves. Por otro lado, las sociedades están conforma- das por individuos cuyas relaciones sociales están basadas en la eficiencia y las relaciones contractuales capitalistas. Es desde esta idea de sociedad o Gesellschaft que Webber (1968) postuló posteriormente que emergen las fuerzas racionales capitalistas del mercado que deshumanizan a las comunidades.

Así, esta construcción dualista de comunidades (premodernas) y sociedades (modernas) sentó las bases para la supuesta distinción entre las economías comunitarias y las de mercado como dos esferas separadas de la economía. De hecho, según Gudeman (2001), lo que hoy se entiende como economía solo considera la parte del mercado dentro de la economía, donde supuestamente todos los bienes tienen un precio y están disponibles para ser intercambiados. Los procesos económicos que escapan al mercado (postcapitalistas según Gibson-Graham, 2006), no son reconocidos como tales, o son representados como irracionales, externalidades u obstáculos para un sistema supuestamente eficiente.

Esta distinción entre las economías comunitarias y de mercado ha sido desarrollada desde distintas perspectivas teóricas, tales como la distinción que Webber (1968) hizo entre las racionalidades sustantivas y las formales, o Polanyi (1977) entre las economías incrustadas y desincrustadas. Gudeman (2001) menciona que si bien estos estudios se han enfocado en cómo las relaciones sociales, la confianza, reciprocidad, cuidado y respeto influyen en el comercio, han tendido a pasar por alto las verdaderas interacciones y conexiones entre estas dos esferas de la economía, y la manera en que las comunidades persisten y apoyan al mercado y viceversa, complementándose mutuamente. Para él, ambas "economías" son dos sistemas duales paralelos, institucionales y tácticamente interconectados, siempre presentes en relaciones complejas y cambiantes.

A pesar de concordar con Gudeman en que diferentes perspectivas económicas coexisten e interactúan significativamente, su trabajo asume una clara distinción entre las dos esferas de la economía, lo que resulta problemático y no logra superar la trampa dualista que presenta a las economías comunitarias y de mercado como sistemas separados que subordinan a lo comunitario. Desde 
una perspectiva de postdesarrollo, el trabajo en torno a las economías diversas liderado por J. K. Gibson-Graham, presenta por otro lado un marco conceptual que rompe con esa dualidad y esa jerarquía. Ofrece un marco analítico para el estudio de las comunidades no como aisladas ni separadas, lo que permite abarcar la complejidad y el valor de iniciativas que, como Trekaleyin, que expanden la diversidad y creatividad de la economía en su totalidad. Es por ello que en la próxima sección se hace uso de las herramientas analíticas específicas proporcionadas por GibsonGraham, las que resultan particularmente relevantes para el caso de Trekaleyin. Pero al examinar esta iniciativa en particular, en este artículo se adopta también una postura decolonial (Grosfoguel, 2007; Santos, 2003) y se incluye de manera explícita en el análisis a las ontologías mapuche para visibilizar aspectos comúnmente descartados de lo económico, y aportar a la descolonización y diversificación de prácticas e ideas acerca de la economía y el desarrollo, incluyendo el turismo.

\section{Economías diversas}

En su influyente libro Gibson-Graham (2006) propone que entender la economía como un ensamblaje de múltiples e imbricadas prácticas, valores, relaciones, saberes, gente y cosas es crucial. Significa reconocer la existencia real y actual de una multiplicidad de economías que incluyen pero no están limitadas al capitalismo, así como también el hecho de que las economías comunitarias no son necesariamente marginales, locales, o remanentes del pasado. Estas nociones son esenciales para entender las economías diversas del Alto Biobío no como destinadas a la extinción, sino que como parte de la economía en su totalidad, la que al mismo tiempo es transformada y expandida. Así, se abren espacios conceptuales y prácticos para reconocer y aportar a procesos económicos y de desarrollo postcapitalistas y sustentables (Healy, 2009; Healy \& Graham, 2008).

Desde una perspectiva geográfica de postdesarrollo, Gibson-Graham (2005: 6, traducción propia) se ha abocado a crear un "discurso de diferencia económica" para amplificar las posibilidades de imaginar y practicar la economía y el desarrollo de manera diferente, más allá de la globalización neoliberal y el capitalismo, y contribuir a una "política de innovación económica" que "aumente las posibilidades de experimentación económica". Pero estudiando las economías comunitarias, ellas llaman a ser cuidadosos con intentar definir, aunque sea vagamente, lo que la economía comunitaria es y no es, para evitar crear representaciones normativas que valore ciertas prácticas y excluya otras. En cambio, se han enfocado en la noción de la "comunalidad" de ser, el que no siendo ser un concepto normativo, abre espacios para negociaciones éticas (Roelvink y GibsonGraham, 2009). Esto significa que más allá de cualquier fantasía acerca de la existencia de economías comunitarias perfectas y libres de "negociaciones, luchas, incertidumbre, ambivalencia y decepción" (Gibson-Graham, 2006: 98-99, traducción propia), ella enfatiza el "existir en común".

Así, para Gibson-Graham las economías comunitarias incluyen todas

\begin{abstract}
“aquellas prácticas económicas que sustentan la vida y mantienen el 'bienestar directamente' ... que 'distribuyen' los 'excedentes' para el mantenimiento material y cultural de las comunidades y activamente hacen y comparten los 'comunes'" (Gibson-Graham, 2005: 16, traducción propia, énfasis en el original).
\end{abstract}

Juntas, todas estas ideas y prácticas constituyen economías diversas, que incluyen pero van más allá del capitalismo y el mercado (Healy, 2009). Esta idea es ilustrada en la imagen de un iceberg (Gibson-Graham et al., 2013) en el cual las transacciones formales de mercado, empleo y las empresas capitalistas se ubican en la punta visible,

"sostenidas en un gran número de sumergidas pero sustentadoras transacciones alternativas y no de mercado, trabajo alternativamente pagado y no pagado, [y] empresas capitalistas alternativas y no capitalistas" (Gibson-Graham \& Roelvink, 2009: 329, traducción propia).

Entender la economía en estos términos tiene dos consecuencias importantes. En primer lugar, hace creíble la existencia y emergencia de múltiples economías, y cuestiona la hegemonía del capitalismo como la forma 
"natural" de la economía. Así, la economía deja de ser algo singular, irrefutable, fijo, para ser entendida como una pluralidad en constante construcción (Graham \& Cornwell, 2009). Y segundo, expande las posibilidades de entender, inspirar, construir y apoyar vías de desarrollo alternativas y contrahegemónicas, donde la innovación económica puede proliferar basada en la diversidad y la complejidad (Gibson-Graham, 2006).

Reconociendo que las perspectivas capitalocentristas tienden a ver todas las actividades como capitalistas o contenidas dentro del capitalismo, y que por lo tanto tienen el efecto paralizante de volver cualquier alternativa como imposible o utópica, es importante tener cuidado en cómo evaluamos iniciativas económicas, ya que podemos terminar reforzando estas posiciones capitalocentristas que asumen que el capitalismo es algo universal, monolítico e imperturbable (Gibson-Graham, 2006). Así, nuestro trabajo puede llevar a socavar, volver no creíble o invisibilizar las maneras en las que la gente está actualmente reconstruyendo economías diversas (Cameron et al., 2014), como es el caso de Trekaleyin.

Para superar estas actitudes, GibsonGraham y Cameron (2007) hacen hincapié en la importancia de adoptar una "postura afectiva" que nos permita pensar y poner en práctica otras posibilidades, y que más que juzgar, busque reconocer, y apoyar ejemplos inspiradores desde posiciones reflexivas, afirmativas y prácticas. Esto implica entender estas experiencias, más que como eventos fijos y definitivos, como experimentos en proceso de construcción, comprendiendo que más que evaluaciones y mediciones con criterios externos lo que se necesita es reflexión y estímulos para mejorar su desarrollo (GibsonGraham, 2008). Más allá de romanticismos, una postura positiva y afectiva no genera automáticamente soluciones, pero ciertamente puede fortalecer la resiliencia de economías locales y conlleva tomar la decisión ética y política de afirmar que el "el deseo por una economía más justa no es nunca completamente suprimido" (Gibson-Graham \& Cameron, 2007: 24, traducción propia).

En la siguiente sección, utilizando como herramienta de análisis el marco conceptual desarrollado por Gibson-Graham (2006), examinaré la configuración de las economías diversas de las comunidades involucradas en Trekaleyin, para después, en el apartado que le sigue, abocarme a la consideración de cómo aspectos ontológicos influyen en estas economías y el trabajo de Trekaleyin.

\section{Trekaleyin y las economías diversas del Alto Biobío}

Gibson-Graham ha desarrollado un marco conceptual con el cual explorar las economías diversas, en el cual se le otorga igual valor a todas las actividades y lógicas económicas desde una perspectiva postcapitalista, es decir, en la que el capitalismo es un elemento más y no el centro de la economía. Este marco analiza cómo las formas de producción (capitalistas, alternativas y no capitalistas), las transacciones (de mercado, alternativas y no de mercado), y el trabajo (remunerado, alternativamente remunerado y no remunerado), conforman economías que mantienen el bienestar de las comunidades directamente, con el que a continuación examinaré las economías pewenche involucradas en Trekaleyin.

En la economía de las comunidades pewenche, la continuidad territorial y el acceso y movimiento libre son esenciales para las actividades económicas. Una particularidad de estas economías pewenche, que las distingue no solo de las no mapuche sino incluso entre las economías mapuche en general, es la trashumancia anual basada en el uso cíclico de las diferentes zonas altitudinales de sus territorios cordilleranos. Así, las economías pewenche se basan en el movimiento según las estaciones del año. En términos productivos, durante otoño e invierno, los miembros de las comunidades ocupan las tierras bajas en los valles cordilleranos o invernadas, donde están ubicadas las viviendas permanentes y cae menos nieve. En la primavera y el verano, por otra parte, familias enteras o miembros de estas se trasladan a las tierras más altas o veranadas, donde los pastizales permiten alimentar al ganado al permanecer verdes durante el verano. La crianza de ganado es la principal actividad económica de la mayoría de las familias pewenche para acumular riqueza, y es uno de los motivos por los cuales se desplazan a través de distintas zonas ecológicas durante el año. Es por 
ello que la reducción de las tierras controladas por las comunidades como consecuencia de su apropiación por parte de terceros desde mediados del siglo diecinueve, ha acarreado importantes consecuencias para las economías pewenche (Molina y Correa, 1998). El ganado, que incluye ganado mayor como vacuno y equino, así como ganado menor incluyendo ovinos y caprinos, provee de carne, leche y fuerza para el trabajo a las familias, así como también lana y cuero para distintos tipos de artesanías, otra actividad económica importante. Pero en las tierras altas existe otro elemento crucial no solo para las economías pewenche, sino también para las identidades y ontologías que las sustentan. Es aquí donde se encuentran los bosques de pewén o araucaria (araucaria araucana), elemento sagrado en la cultura mapuche-pewenche y que define no solo aspectos especiales de sus actividades económicas y dieta, sino que también su identidad de forma intrínseca (pewén-che significa la gente del pewén). El ngülliw o piñón, fruto de la araucaria, se recolecta en estos bosques, al igual que otros productos como hongos (digüeñes y changles), semillas, plantas medicinales y leña.

En las invernadas, alrededor de las casas se plantan huertas con vegetales y árboles frutales, y se crían aves y cerdos. Un poco más alejadas se plantan cultivos como el trigo y las papas. Sin embargo la agricultura más extensiva ha ido disminuyendo debido a factores como el crecimiento de la población y las regulaciones impuestas por Corporación Nacional Forestal (CONAF) que prohíben la roza (Molina y Correa, 1998). En estos sectores de invernadas se mantiene el ganado durante el invierno, y la apicultura ha sido adoptada exitosamente en las últimas décadas.

El turismo es una actividad más bien reciente e incipiente entre las comunidades. Comenzó como tal a principio de la década de los noventa, y comprende actividades tanto en los espacios de invernadas (i.e. sitios de camping, cabañas) como en las veranadas, donde muchas de las cabalgatas se desarrollan. Pero junto con estas actividades económicas productivas, las comunidades desarrollan también actividades reproductivas tales como las tareas del hogar, el cuidado de niños, ancianos y enfermos, y la edificación y mantención de construcciones, entre otros.

En general, entonces, la producción en estas comunidades, como en muchas de las comunidades rurales mapuche en Chile, se puede identificar como diversa en tanto no es realizada principal ni exclusivamente a través del mercado (COTAM, 2003b). Más bien, se enfoca en la reproducción de las familias y comunidades, y gran parte de lo que se produce se consume dentro de ellas.

En el caso de las transacciones, se reconoce también una matriz diversa, ya que existen distintos tipos de intercambios entre los que el mercado es solo uno más. Con respecto a productos vendidos a través del mercado (utilizando dinero), estos incluyen los excedentes de piñones, hongos y otros productos forestales que se venden en zonas urbanas, la miel que en general se vende a intermediarios, que al igual que las artesanías, se venden también a turistas o en ferias en la región. La ganadería, principal fuente de dinero en efectivo para muchas familias, se tiende a vender a través de intermediarios o en menor medida a visitantes a las comunidades. La ganadería se usa también como una forma de ahorro, y para comprar bienes como harina de trigo, yerba mate, y azúcar entre otros. Así, como ha sido también identificado en comunidades mapuche en otras áreas (COTAM, 2003b), aquí los excedentes son generados no solo en el sentido capitalista (dinero), ya que se utilizan también para mantener una reserva de recursos (semillas, animales, herramientas, dinero) para hacer frente a las incertidumbres, los tiempos difíciles, las ceremonias y eventos sociales, circunstancias especiales como viajes, funerales o enfermedades, y para mantener y mejorar los niveles de vida. De hecho, la manera de acumular y utilizar lo acumulado también tiene particularidades dentro de las comunidades mapuche, involucrando la capacidad de compartir y aportar a instancias comunitarias. Por ejemplo, se ha identificado que la figura del übmeh no solo se asocia con una persona que posee muchos bienes y potencia económica, sino que también que con ser una persona íntegra que demuestre capacidad de dar y compartir, en especial en ceremoniales y espacios de congregación (Quidel et al., 2007). 
Aparte de las transacciones de mercado, productos como las hortalizas, los cultivos, animales, artesanías, leña y piñones son principalmente consumidos por cada familia, intercambiados, compartidos y/o regalados, entretejidos en las relaciones y redes que articulan a los miembros de las comunidades. Estas redes incluyen, entre otros, lazos familiares, amistades y padrinos. Pero estas transacciones no capitalistas pueden incluir elementos culturales particulares, ya que como indica Ñanculef (2015), el txafkintu o intercambio dentro de las comunidades mapuche, no es solo entendido como un intercambio económico, sino que como parte de un entramado social más amplio. Por ello, para él estos intercambios no pueden ser reducidos a relaciones comerciales, sino que se trata más bien de "una reciprocidad de sentimientos", una acción que conlleva la rearticulación de conexiones, relaciones y saberes particulares. Así por ejemplo, el piñón es usado por cada familia en forma cotidiana, pero es también compartido a través del txafkintu y usado de manera colectiva en ceremonias. Pero no olvidemos que los piñones son algo más que una fuente de alimentos, intercambio o dinero en efectivo (cuando son vendidos en las ciudades), al ser un elemento sagrado e identitario por excelencia, que incluye relaciones profundas y complejas no solo entre personas, sino que también con el territorio, las cordilleras, bosques y seres sobrenaturales.

El uso de espacios y recursos comunes también forman una matriz importante para la cooperación y el mantenimiento económico de las comunidades (Molina y Correa, 1998). Por ejemplo, en las comunidades del valle del Queuco (a excepción de Pitril, la única comunidad que posee títulos de propiedad individuales en vez de comunitarios), las veranadas y bosques de araucarias son propiedad de toda la comunidad (propiedad colectiva de la tierra), pero distintas áreas son usadas por diferentes familias designadas de acuerdo a líneas de parentesco. Este uso por distintas familias establece relaciones de apoyo, cuidado y responsabilidades, que se toman en cuenta también al decidir a dónde se llevan turistas en cada viaje.

Por último, en relación al trabajo en el valle del Queco, hay diferentes formas de remuneración que incluyen pero trascienden el trabajo asalariado. Las actividades económicas están diferenciadas de acuerdo a la edad y género de las personas, y en muchos casos son remunerados de forma alternativa (a través de favores $u$ otros) o no remunerados. Entre las familias y vecinos existen redes y prácticas de apoyo para ayudar con las actividades que requieren más mano de obra, tales como la "trilla" y los cultivos extensivos de siembra y cosecha, que por lo general son devueltos con favores posteriores o fiestas. Trabajos no remunerados o voluntarios son también desempeñados en distintas circunstancias, como por ejemplo en las preparaciones para los Nguillatunes y la construcción de ramadas para estas ceremonias. También dentro de esta categoría caben todas aquellas actividades de cuidado y mantención que se realizan de forma cotidiana tales como cocinar, cuidar a niños y ancianos, enseñar a través de generaciones, y otras.

Trabajos remunerados son generalmente desarrollados fuera de las comunidades en una gama de actividades. Es común que jóvenes y adultos trabajen en empleos temporales en agricultura, construcción o servicio doméstico. Otros, de acuerdo a su nivel de educación formal, trabajan en instituciones del Estado y servicios de salud o educación, organizaciones no gubernamentales o empresas privadas. Muy rara vez el trabajo es remunerado entre miembros de las comunidades, y cuando esto ocurre es por trabajos específicos como la construcción. El turismo se ha convertido así en otro caso de trabajo pagado en las comunidades, donde los guías y cocineros son remunerados de acuerdo a su trabajo individual. Esto resulta un tema complejo que ha generado en ciertos casos envidias, tensiones y controversias entre miembros de las comunidades, y que ha requerido ser cuidadosamente considerado.

Pero el turismo es más que una simple forma de trabajo asalariado. Similarmente a lo que Gibson-Graham y otros han identificado en numerosos casos de economías comunitarias (Cameron \& Gibson, 2005), el turismo en Alto Biobío está informado por una diversidad de aspiraciones y entrelazado con variadas relaciones alternativas $y$ no capitalistas. Por ejemplo, examinando las economías diversas de Jagna, Filipinas, Gibson-Graham (2005) ha identificado cómo 
el trabajo está enredado en relaciones de compartir y cooperación a través de relaciones recíprocas, intercambios, donaciones voluntarias de trabajos, y pagos en especies. De forma similar, Trekaleyin está influenciado por las redes familiares, relaciones sociales y tejidos de favores, compromisos y otras formas de remunerar el trabajo entre miembros de las comunidades, lo que cuestiona sus motivaciones como simplemente individualistas y orientadas al lucro. Por ejemplo, la elección de quien es o no incluido como trabajador pagado en el turismo está basado no solo en los roles predeterminados de las personas, sino que también en sus necesidades y circunstancias particulares, tales como las dificultades que algunas familias estén enfrentando o preparaciones para eventos especiales (i.e. bodas, viajes, estudios). Además, trabajar en turismo suele estar vinculado a favores anteriores o relaciones familiares y sociales más amplias, y desde 1998 los miembros de las comunidades han expresado su preferencia por trabajar colectivamente en el turismo en lugar de individualmente (Molina et al.,1998). De hecho, aunque el trabajo se paga de acuerdo al trabajo individual, con frecuencia involucra a toda la familia, o incluso a veces grupos de familias, que apoyan y participan en la actividad. Los campings, por ejemplo, a pesar de ser empresas familiares, por lo general también involucran a vecinos a los que se les pide que vengan a ayudar y se les paga en formas que incluyen, pero van más allá del dinero. Asimismo, en las "veranadas" donde las cabalgatas son desarrolladas, familias que podrían no estar directamente involucradas en Trekaleyin se unen para compartir con los turistas, y en muchos casos se hacen acuerdos para que ellos puedan también participar en el turismo, por ejemplo, preparando alimentos o vendiendo animales para los asados.

Todas estas formas de trabajo "asalariado" hacen hincapié en la importancia de los valores de reciprocidad y redistribución entre las comunidades, y en la naturaleza colectiva del turismo en el valle del Queuco. Pero también ocurre que personas se involucran en turismo sin ser remunerados por razones que incluyen, entre otros, deseos de aprender y capacitarse, transmitir conocimientos, monitorear lo que se hace o simplemente divertirse. Por ejemplo, es común entre los guías de Trekale- yin llevar a hijos o sobrinos jóvenes con ellos a las cabalgatas de forma gratuita, como una forma de capacitarlos en el turismo y de tener un apoyo extra. Personas mayores o sabias de algunas comunidades también se unen a grupos de turistas sin ser pagados como una forma de interactuar con los turistas y compartir sus historias y conocimientos, así como supervisar la forma en que se está llevando a cabo la actividad. Y otros se involucran con los turistas por diversión, ya que disfrutan de conocer gente nueva y encuentran agradable su compañía.

En conclusión, al examinar las economías de las comunidades involucradas en Trekaleyin desde una perspectiva amplia, y ver cómo el turismo se entrelaza en ellas, se puede distinguir que articulan elementos que incluyen, pero van más allá del capitalismo. Se trata, entonces, de economías diversas en las que el turismo, siguiendo las palabras de Gibson-Graham y Cameron (2007: 21, traducción propia), es una iniciativa "cuyo 'negocio central' no es maximizar el beneficio privado, sino que producir el bienestar de la comunidad directamente". Para ello, como se ha demostrado, se entretejen formas de producción, transacciones y trabajo que no se reducen al capitalismo, y en las que este ni siquiera está al centro.

Desarrollado como una actividad económica complementaria en las comunidades, el turismo está formado por y articulado con las economías de mercado y el dinero, así como otras formas y redes de producción, reproducción, transacciones y trabajo. Está informado por la coexistencia, interdependencia y fricciones entre familias, personas y comunidades, las conexiones de linajes y lugares, recursos comunes como las veranadas, bosques y senderos, y elementos de propiedad individual y familiar como caballos, carpas y sitios de camping. Junto con apuntar a que las prácticas capitalistas son solo una parte en ensamblajes económicos más amplios en las comunidades como Gibson-Graham (2006) sugiere, esta diversidad económica deja entrever también que hay aquí otros valores y ontologías que influyen en las formas particulares de ser y saber en la economía. En la siguiente sección exploraré en mayor detalle las formas en que estas ontologías informan la vinculación de los miembros de las comu- 
nidades con el turismo, y la importancia de vislumbrar estos elementos para avanzar en la descolonización del saber y la economía.

\section{Dimensiones ontológicas y lo económico}

Junto con el reconocimiento de su diversidad, una visión más exhaustiva de la economía puede también cuestionar nociones occidentales de la modernidad y la colonialidad, socavando sus presupuestos universalistas (Escobar, 2005; Prada, 2010). Escobar (2005) plantea que la transición a la modernidad en Europa occidental se caracterizó por el auge de la economía capitalista y el individuo, acompañado por el despertar del expansionismo europeo y el colonialismo. Desde entonces, según él, el capitalismo, la economía de mercado y sus prácticas culturales asociadas han sido impuestos al resto del mundo, en particular a partir de la segunda mitad del siglo XX a través de la idea de desarrollo, llevando a la subordinación e invisibilización de otras formas de pensar, vivir y construir economía. Esta imposición, sin embargo, ha encontrado numerosas formas de resistencia y creatividad con las que personas, comunidades e incluso países se han relacionado con el capitalismo. Pero este encuentro no es algo menor, ya que según De la Cadena (2009) conlleva un "choque de ontologías" que implica la negociación de la pluralidad de manera profunda. Al respecto, Escobar (2010) ha sugerido que los movimientos indígenas y sociales contemporáneos en América Latina están abordando directamente la defensa y el reconocimiento de la coexistencia de diversas ontologías, en las cuales están en juego diferentes concepciones de la vida y significados, prácticas y relaciones económicas. Blaser (2009) usa el concepto de ontologías políticas para enfatizar estos procesos en los que distintas ontologías entran en conflicto y son negociadas. Para él, entonces, no se trata solo de defender derechos o acceso a ciertos recursos, sino que también de cómo estos elementos son entendidos desde perspectivas ontológicamente distintas, que son supeditadas a concepciones modernas occidentales. Escobar (2010) identifica entre los aspectos ontológicos en disputa, saberes y actores silenciados y subordinados que desafían importantes pilares de la modernidad, el desarrollo y la economía occidentales, tales como:

"[la] primacía del ser humano sobre los no-humanos (separación de la naturaleza y la cultura) y de algunos humanos sobre otros (la división colonial de nosotros y ellos); la idea del individuo autónomo separado de la comunidad; la creencia en el conocimiento objetivo, la razón y la ciencia como los únicos modos válidos de conocimiento; y la construcción cultural de 'lo económico' como una entidad autorregulada fuera de las relaciones sociales" (Escobar, 2010: 9, traducción propia).

De la Cadena (2009) menciona que estas otras ontologías están a menudo al centro de las propuesta indígenas, articulando y sacando de las "sombras" maneras distintas de entender las relaciones entre personas y los demás seres. Así, se crean espacios de negociación "fuera de lo común" (p. 162) ya que actores inusuales, tales como ancestros, espíritus, y otros seres no humanos, son incorporados en negociaciones políticas y económicas. Por lo tanto, resulta urgente hoy reconocer estas disputas ontológicas como tales para avanzar en los procesos de descolonización, desestabilizando el consenso moderno que ha excluido prácticas y saberes indígenas de lo económico y político, relegándolos a los ámbitos rituales, religiosos o de la superstición.

Ontologías mapuche-pewenche, la economía y el turismo. La experiencia de Trekaleyin

Se ha reconocido que en la economía de las comunidades mapuche elementos culturales y sociales constituyen una matriz subyacente que guía las decisiones económicas e influyen en la producción, circulación y distribución de bienes y servicios (COTAM, 2003a; Marimán et al., 2006; Ñanculef, 2015). Así también, en el concepto de "mundo mapuche" acuñado por organizaciones e intelectuales mapuche, se involucran memoria, presente y futuro al entrelazar principios, valores y espiritualidades que ordenan discusiones en torno a la economía, el desarrollo y el territorio (Hernández, 2003; Marimán, 2005). Sin embargo, tomar en cuenta las on- 
tologías mapuche-pewenche en serio en la economía es un acto político y controversial. Confronta a quienes sugieren que la cultura y el pueblo mapuche han desaparecido, son irrelevantes o arcaicos. Sin embargo, en este artículo se busca explorar explícitamente la influencia que estas tienen en la economía, haciéndolas visibles y resaltando su ininterrumpida persistencia para contribuir a la descolonización del pensar económico en general, y de la realidad en comunidades mapuche en particular. Además, es una forma más de examinar en la práctica las formas en que aspectos ontológicos están siendo actualmente negociados en las propuestas indígenas.

Según el kimüm (sabiduría) mapuche, el küme felen es el fin último de la vida y significa bienestar en armonía. Incluye la tranquilidad y el bienestar de las personas con ellas mismas, sus territorios, el entorno natural y con las distintas fuerzas o newenes del territorio (COTAM, 2003a; Ibacache et al., 2002). Por lo tanto, el küme felen se refiere al bienestar en términos generales, involucrando a seres naturales y sobrenaturales, y contiene la idea de küme mongen o calidad de vida (Reiman, 2007 en: Tricot, 2009). Involucra también aspectos económicos, en el sentido de poseer suficientes recursos materiales para vivir bien, no solo a nivel individual sino que también colectivo, y por ello se refiere también al bienestar y equilibrio social (Ibacache et al., 2002).

Para mantener el bienestar o küme felen, es importante mantener el balance y armonía entre las fuerzas positivas y negativas a través de una correcta conducta (nor felen), siguiendo los lineamientos éticos dictados por el Az Mapu, o las reglas que rigen toda los aspectos de la vida (Chihuailaf, 1999; Marileo, 2002). A pesar de ser específico para distintos territorios, el Az Mapu posee algunos principios generales o valores tales como reciprocidad, respeto, admiración, solidaridad, el cuidado por los otros, y la defensa de uno mismo y el colectivo (COTAM, 2003a; Rupailaf, 2006). Así, activistas mapuche han reconocido que hoy la economía en las comunidades mapuche está aún basada en los principios de bienestar integral (küme felen), es regulado por el Az Mapu, y está centrado en torno al lof o comunidad (Organizaciones Territoriales Mapuche, 2006).
Esta sabiduría y valores influyen y son movilizados en Trekaleyin de diversas maneras. Trekaleyin es una iniciativa turística que busca el bienestar de las comunidades, motivada por deseos y aspiraciones que incluyen pero van más allá de lo económico. A pesar de que es importante para los involucrados, el ingreso que ha traído Trekaleyin no es demasiado alto en términos absolutos. Sin embargo, no existe la intención de maximizarlo a cualquier costo, y las maneras en que el turismo es planificado y limitado está más bien ligado a promover el balance entre personas, comunidades, lugar, naturaleza y las diferentes fuerzas o newenes que los rodean, resonando así con nociones más amplias del küme felen y el Az Mapu.

Así, por ejemplo, la manera en la que Trekaleyin opera reconoce la interdependencia y gratitud hacia otros seres y fuerzas, y la necesidad de respetarlos. Así, mantener el nor felen o conducta adecuada para no perturbar a las fuerzas (newenes) y espíritus (ngens) presentes en el territorio es crucial y delinea la manera de relacionarse con los turistas, como lo expresa Leonel, un guía:

"Cuando yo veo por primera vez a un turista, por la forma que se expresa uno ve si es gente respetuosa. Porque yo siempre que salgo con gente a los senderos antes les doy una recomendación. Para empezar les digo que existe la cordillera, los volcanes, el agua, el estero, el río, el lago, y que todos ellos tienen dueño, tienen ngen, que no están solos. Si bien son invisibles existen, y nosotros como pewenche creemos en eso. Les digo que nadie puede andar haciendo desorden, y así uno va viendo en el camino si están cumpliendo lo que uno les advirtió o no. Por eso es difícil en el camino ir conversando más como dicen las encuestas de los turistas a veces, porque uno tiene que ir con respeto y además fijarse en la gente, ver cómo se van portando.

... Pero también es lindo, porque uno puede mostrar la riqueza, todas las cosas, los árboles, la montaña, las cascadas, todo lo que "el que está arriba" nos dio. Sentirlo y valorarlo, dar las gracias y compartirlo. Porque para mí sobre todo en los árboles yo encuentro siempre sabiduría y fuerza. Cuando tengo problemas me sien- 
to debajo y siento como que me hablan, como que si lloro ellos también lloran conmigo. Está esa fuerza que me calma, me ayuda a seguir adelante".

Mantener este respeto y consideración por elementos naturales y sobrenaturales, entonces, es primordial. Puede llegar a ser más importante que cumplir con lo que se sugiere en encuestas con turistas como conversar más en las cabalgatas, si lo necesario es mantener el respeto, ser cuidadoso y observar. La decisión de seguir las reglas del Az Mapu también influye en los límites que los miembros de Trekaleyin ponen en los eventos y actividades que se comparten o no con los turistas. Así, como Eusebio menciona a continuación, se ha decidido no involucrar a los turistas en actividades que potencialmente podrían aumentar significativamente sus ingresos, por respeto a los espacios sagrados y a la decisión voluntaria de cuidar el equilibrio y armonía social dentro de las comunidades. Esto es lo que Eusebio mencionó:

“Nosotros no vamos a llevar los turistas a un Nguillatún porque eso es sagrado, es un lugar de ceremonia. Aunque podría vender harto como en otras partes, pero nosotros decidimos que no. No queremos folclorizarnos porque hay que respetar lo nuestro, la cultura, lo sagrado, por lo hay que poner límites, reglas".

Constantemente, entonces, al incorporar elementos del nor felen y Az Mapu en el desarrollo de la actividad turística distintas ontologías y prioridades son negociadas. Elementos como el dinero, el mercado y conexiones con turistas se entrelazan con otros cruciales elementos también importantes en la búsqueda del küme felen. Así, este bienestar en términos amplios no implica perseguir un aumento constante de los ingresos, crecer a cualquier costo o tener más y más proyectos, sino más bien, y tal como lbacache et al. (2002: 14) mencionan, se concibe como tener suficiente para tener un "buen pasar, una buena calidad de vida" siguiendo las normas del Az Mapu.

Por lo tanto, si entendemos la economía como diversa y articulando diferentes ontologías, las ganancias pueden ser también entendidas como diversas e híbridas. En el caso de Trekaleyin, estas ganancias varias incluyen el dinero, claro está, pero también relaciones de cuidado, respeto y bienestar en términos amplios y reconociendo la relevancia de actores que van desde los humanos a seres naturales y sobrenaturales que, por ejemplo, cuidan y habitan esteros y bosques, y están al centro de ceremonias como el Nguillatún. Pero también se buscan otras "ganancias" a través de Trekaleyin a nivel colectivo y territorial, enraizadas en las ontologías pewenche así como en sus luchas y contexto histórico y político. Para ejemplificar estas otras utilidades colectivas y territoriales examinaré a continuación la experiencia de Eusebio, uno de los fundadores de Trekaleyin.

Eusebio ha dedicado años de trabajo y esfuerzo a fortalecer Trekaleyin, a pesar de recibir muy pocos ingresos a través de su trabajo en Trekaleyin. Desafiando la idea del "homo economicus" que solo trabaja en su propio beneficio, e ilustrando las formas de trabajo y ganancias diversas más allá de las capitalistas, él no trabaja como guía ni coordinador de cabalgatas, sino que ocasionalmente otorga alimentación a turistas ya que su negocio turístico está orientado a otro tipo de público (más bien familiar y de descanso). Sin embargo, Eusebio está convencido de que el desarrollo del turismo beneficia a todos en el área, ya sea directa o indirectamente, y que Trekaleyin ayuda a promover el valle del Queuco como destino. Pero más importante aún, él cree que el tener una organización pewenche fuerte trabajando en turismo en el área, que se ha vuelto crecientemente atractiva para gente de afuera, puede prevenir la llegada de operadores turísticos privados interesados solo en "usar" el territorio, vinculándose con los miembros de las comunidades solo como mano de obra relativamente barata y llevando a su subordinación y vulnerabilidad. O al menos, opina Eusebio, tener una organización como Trekaleyin puede hacer más complicada este tipo de intervención privada y ayudar a mejorar las posibilidades de negociar de las comunidades, asegurándose un trato justo y control.

Pero además Eusebio cree firmemente que el turismo es una buena manera de reforzar la cultura pewenche, la defensa de su control territorial y la búsqueda de alternativas de desarrollo elegidas y manejadas por las comu- 
nidades. En primer lugar, aporta a mantener vivos y visibles elementos culturales mapuche-pewenche, a autorrepresentar su cultura, aumentar la valoración de su identidad, y a enseñarle a gente de afuera acerca de su riqueza cultural. En segundo lugar, mantiene activas y fuertes instancias de coordinación y colaboración a nivel territorial en el Valle del Queuco, y promueve considerar colectivamente el manejo y control de iniciativas en el territorio. $Y$ en tercer lugar, según él el turismo puede ayudar a buscar alternativas de desarrollo tales como fuentes laborales para que especialmente las generaciones más jóvenes no tengan que emigrar a las ciudades.

Por lo tanto para Eusebio Trekaleyin, como una iniciativa colectiva, puede aportar al fortalecimiento de la autonomía y organización de las comunidades, al mismo tiempo que a mejorar sus condiciones materiales. Así, el compromiso de Eusebio en el desarrollo del turismo no emerge solamente de un interés monetario. En cambio surge de una combinación de intereses que incluyen mayores ingresos hasta cierto punto, pero intercalado con aspiraciones culturales, territoriales y políticas. Articula así elementos que aportan al küme felen en su dimensión colectiva y de equilibrio social y territorial. Por ello, estos objetivos, y las maneras en que se maneja el turismo en Trekaleyin, está ligado directamente a valores y regulaciones contenidas en el Az Mapu como el respeto, la solidaridad, la autorregulación, el cuidado, y a la intención de mantener una conducta adecuada (nor felen), donde las personas están motivadas a actuar de manera sabia, responsable y generosa. Esto significa que el desarrollo del turismo involucra procesos complejos y negociados ligados al dinero, la reciprocidad, respeto, cuidado, balance, y otros valores, en los que no solo están involucrados quienes participan del turismo, sino que también ancestros, generaciones futuras, las comunidades en su totalidad, y actores naturales y sobrenaturales.

\section{Conclusión}

Al reconocer la gama de valores, seres, prácticas y expectativas que entretejen y dan forma a Trekaleyin y los ensamblajes económicos diversos de las comunidades, es nece- sario volver a preguntarse acerca de cómo se puede entender la relación entre los espacios económicos comunitarios y de mercado ise pueden separar? Y si es así, ¿cómo y dónde trazamos los límites?

Estudios recientes han considerado las múltiples dimensiones y conexiones del turismo en comunidades indígenas, y cómo estas lo articulan es sus sistemas económicos y socioculturales particulares. Pero a pesar de su contribución a una mejor comprensión del turismo y las economías de estas comunidades, estos estudios han tendido a mantener intacta la distinción entre economías del mercado y comunitarias como claramente diferenciables. Por ejemplo, al estudiar la experiencia de una comunidad indígena en Ecuador, Ruiz-Ballesteros y Hernández-Ramírez proponen que el turismo es un fenómeno multidimensional e híbrido, ya que es "otra de las actividades desarrolladas por las comunidades, tanto en términos de la lógica del Mercado como de la lógica de las relaciones comunitarias de reciprocidad, en una especie de economía dual" (2010: 206 traducción propia). Esta división entre dos supuestos ámbitos separados de la economía también se encuentra, aunque de manera distinta, en el trabajo de Bunten (2010) y Spiller et al. (2011), y está claramente influenciada por una comprensión dualista de la economía. Esta diferenciación, sin embargo, es problemática en términos conceptuales y prácticos al examinar la experiencia de Trekaleyin. No solo no reconoce que la economía a todo nivel, y no solo entre los pueblos indígenas, es siempre híbrida, diversa e influida por elementos culturales, valores y otros (Amin \& Thrift, 2007; Gibson-Graham, 2006), sino que además refuerza representaciones estereotípicas y uniformes de las comunidades (Blackstock, 2005), así como nociones estáticas de lo tradicional versus lo (occidental) moderno (Quijano, 2008).

Al mirar cuidadosamente la experiencia de Trekaleyin, sin embargo, la distinción entre las economías comunitarias y de mercado se vuelve borrosa y deja de ser tan evidente y predeterminada. Como ha sido demostrado a lo largo de este artículo, el turismo está lejos de ser una actividad solamente capitalista. En el Valle del Queuco está entrelazado en un entramado constituido por elementos ca- 
pitalistas y no capitalistas, que incluyen una gama de actividades productivas y reproductivas, transacciones y formas de remunerar (o no) el trabajo. Incluye también distintas relaciones, emociones, valores y ontologías que no pueden ser fácilmente separadas y están en constante negociación. Esta multiplicidad permite reconocer la existencia de "una fina capa de actividad económica capitalista sustentada por una gruesa malla de prácticas y relaciones en torno al dar, compartir, prestar, voluntariar y reciprocar el trabajo individual y colectivo" (Gibson-Graham, 2005: 16, traducción propia).

Este artículo, al reconocer esta hibridez y las particularidades de articulaciones económicas diversas, busca presentar una comprensión más detallada, descolonizada y realista de la economía. Para ello es importante reconocer que más que dos esferas separadas, la economía comunitaria y de mercado son inseparables y coconstituyen articulaciones económicas particulares, cambiantes y negociadas a través de procesos constantes de acomodación y creatividad. En otras palabras, es importante reconocer el carácter real, performativo, híbrido y negociado de los ensamblajes económicos, lo que tiene implicaciones que van mucho más allá de lo "local" (Amin \& Thrift, 2004; Marston et al., 2005).

En este artículo desde una postura afectiva positiva que más que juzgar busca reconocer y apoyar iniciativas para continuar construyendo otras economías posibles (GibsonGraham \& Roelvink, 2009), ha buscado visibilizar e incluir conocimientos, prácticas y formas de ser que suelen borrarse de los discursos económicos o incluso son negados por completo. Se ha argumentado que el turismo, cuando se hace con una atención cuidadosa y respetando las reglas del Az Mapu pewenche, puede contribuir al küme mongen o buena vida, donde, según Ibacache (1997), el desarrollo, la equidad, la organización y autodeterminación son elementos claves. Para Trekaleyin esto implica decidir cuidadosamente qué y cómo se comparte con los turistas, así como construir oportunidades para permanecer en sus territorios, fortalecer su bienestar y coordinación, controlar la forma en que son representados, y la perpetuación de su cultura y la soberanía dentro de marcos ontológicos propios.

Así, en términos más amplios, este artículo ha también contribuido a visibilizar la contribución de saberes mapuche-pewenche hacen a debates en torno a la economía y el desarroIlo. Al entender el bienestar como un balance social, físico, espiritual y medioambiental, en el que están involucrados los individuos, las familias, comunidades y todo el tejido social, estos saberes aportan una visión más holística y compleja (Rupailaf, 2006). Comunidades, territorios, identidades, y kimün mapuche, son claves en procesos de descolonización y de involucrarse creativamente con iniciativas económicas, y deben ser consideradas seriamente al pensar en qué se entiende por bienestar y desarrollo, y cómo consideramos las opciones para alcanzarlo.

En general, entonces, considerar los ensamblajes económicos diversos de las comunidades nos ha permitido avanzar hacia una perspectiva postcapitalista (Gibson-Graham, 2006) y decolonial (Grosfoguel, 2007). Así como el postdesarrollo insinúa la posibilidad de un tiempo en que el desarrollo ya no es el principio organizador de la sociedad, el postcapitalismo habla de una situación en la que el capitalismo ya no es la forma hegemónica de la economía (como suele serlo en los marcos capitalocéntricos de la mayoría de las economías políticas) (Escobar, 2010). Así, para Escobar (2010) el postcapitalismo significa el desplazamiento de la hegemonía del capitalismo en la economía, un desplazamiento que no ha de ser alcanzado en el futuro pero que está siendo actualmente construido por medio de experiencias tales como Trekaleyin. Por otro lado, en términos de avances hacia saberes decoloniales, este artículo permite entender cómo agrupaciones indígenas están implícitamente incluyendo sus ontologías particulares en esferas más allá de lo cultural, como en lo económico, medioambiental y relaciones con no indígenas (turistas y otros). Así, se van cambiado los términos de la conversación y de cómo se pueden imaginar alternativas (Blaser \& de la Cadena, 2009), cuestionando entre otros la separación de los humanos y los no humanos, el individuo de lo comunitario, y lo económico de relaciones sociopolíticas más amplias (Escobar, 2010). 


\section{Referencias bibliográficas}

AITKEN, S.C. Community. In: KITCHIN, R. \& THRIFT, N. International Encyclopaedia of Human Geography. Amsterdam/Oxford: Elsevier, 2009, p. 221-233.

AMIN, A. \& THRIFT, N. Introduction. In: AMIN, A. \& THRIFT, N. The Blackwell cultural economy reader. Oxford: Blackwell Publishing, 2004, p. x-xxx.

AMIN, A. \& THRIFT, N. Cultural-economy and cities. Progress in Human Geography, 2007, Vol. 31, Nº 2, p. 143-161.

BLACKSTOCK, K. A critical look at community based tourism. Community Development Journal, 2005, Vol. 40, N 1, p. 39-49.

BLASER, M. Political ontology: Cultural Studies without 'cultures'?. Cultural Studies, 2009, Vol. 23, N 5-6, p. 873-896.

BLASER, M. \& DE LA CADENA, M. Introduction. Journal of the World Anthropology Network, 2009, N 4, p. 3-9.

BUNTEN, A.C. More like ourselves. Indigenous capitalism through tourism. American Indian Quarterly, 2010, Vol. 34, N 3, p. 285312.

BUSHEL, R. y SALAZAR, J.F. Turismo indígena en San Pedro de Atacama, Chile. Sydney: Informe desarrollado para la Fundación Minera Escondida por el Centro de Investigaciones Culturales-Sydney: Univesity of Western Sydney, 2009.

CAMERON, J. \& GIBSON, K. Alternative pathways to community and economic development: The Latrobe Valley Community Partnering Project. Geographical Research, 2005, Vol. 43, No 3, p. 274-285.

CAMERON, J.; GIBSON, K. \& HILL, A. Cultivating hybrid collectives: research methods for enacting community food economies in Australia and the Philippines. Local Environment, 2014, Vol.19, № 1, p. 118-132.

CHIHUAILAF, E. Recado confidencial a los chilenos. Santiago de Chile: LOM Ediciones, 1999.
COMISIÓN DE TRABAJO AUTÓNOMO MAPUCHE (COTAM). Informe de la Comisión verdad histórica y nuevo trato, Volumen III, Tomo 2: Primera parte del informe final de la Comisión de Trabajo Autónomo Mapuche (COTAM). Santiago de Chile: Mapu Küpal Azkunun Zugu. Fundamentos y Manifestaciones del derecho propio mapuche, 2003a.

COMISIÓN DE TRABAJO AUTÓNOMO MAPUCHE (COTAM). Informe de la Comisión verdad histórica y nuevo trato, Volumen III, Tomo 3: Segunda parte del informe final de la Comisión de Trabajo Autónomo Mapuche (COTAM). Santiago de Chile: Transformaciones del sistema económico mapuche a la luz de las políticas estatales, los procesos de integración y la globalización sociocultural, 2003b.

DE LA CADENA, M. Política indígena: Un análisis más allá de "Ia política". Journal of the World Anthropology Network, 2009, Nº 4, p. 139-171.

ESCOBAR, A. Economics and the space of modernity. Tales of market, production and labour. Cultural Studies, 2005, Vol. 19, N² p. $139-175$.

ESCOBAR, A. Latin America at a crossroads. Alternative modernizations, post-liberalism, or post-development?. Cultural Studies, 2010, Vol. 24, N²1, p. 1-65.

FAULCONBRIDGE, J.R. \& HALL, S. ECOnomics and human geography. In: KITCHIN, R. \& THRIFT, N. International Encyclopaedia of Human Geography. Amsterdam/Oxford: Elsevier, 2009, p. 332-337.

GIBSON-GRAHAM, J.K. Surplus possibilities: postdevelopment and community economies. Singapore Journal of Tropical Geography, 2005, Vol. 26, № 1, p. 4-26.

GIBSON-GRAHAM, J.K. A postcapitalist politics. Minneapolis/London: University of Minnesota Press, 2006.

GIBSON-GRAHAM, J.K. Diverse economies: Performative practices for 'other worlds'. Progress in Human Geography, 2008, Vol. 32, N 5, p. 613-632. 
GIBSON-GRAHAM, J.K. \& CAMERON, J. Community enterprises: Imagining and enacting alternatives to capitalism. Social Alternatives, 2007, Vol. 26, Nº 1, p. 20-25.

GIBSON-GRAHAM, J.K.; CAMERON, J. \& HEALY, S. Take Back the Economy: An Ethical Guide for Transforming Our Communities. Minneapolis: University Of Minnesota Press, 2013.

GIBSON-GRAHAM, J.K. \& ROELVINK, G. An economic ethics for the anthropocene. Antipode, 2009, Vol. 41, N 1, p. 320-346.

GRAHAM, J. \& CORNWELL, J. Building community economies in Massachusetts: An emerging model of economic development? In: AMIN, A. The social economy: International perspectives on economic solidarity. London: Zed Books, 2009, p. 37-65.

GROSFOGUEL, R. The epistemic decolonial turn. Cultural Studies, 2007, Vol. 21, Nº 2, p. 211-223.

GUDEMAN, S. The anthropology of economy: community, market, and culture. Malden: Blackwell, 2001.

HEALY, S. Economies, alternative. In: KITCHIN, R. \& THRIFT, N. International Encyclopaedia of Human Geography. Amsterdam/ Oxford: Elsevier, 2009, p. 338-344.

HEALY, S. \& GRAHAM, J. Building community economies: A postcapitalist project of sustainable development. In: RUCCIO, D. Economic Representations: Academic and everyday. New York: Routledge, 2008, p. 291314.

HERNÁNDEZ, I. Autonomía o ciudadanía incompleta. El pueblo Mapuche en Chile y Argentina. Santiago de Chile: Pehuén Ediciones, 2003.

IBACACHE, J. La salud, el desarrollo y la equidad en un contexto intercultural. Uppsala: Working Paper Series 2 Ñuke Mapuförlaget, 1997.

IBACACHE, J.; MCFALL, S. \& QUIDEL, J. Rume Kagenmew Ta Az Mapu. Epidemiología de la trasgresión en Makewe-Pelale. Uppsala:
Working Paper Series 2 Ñuke Mapuförlaget, 2002.

MARILEO, A. Mundo Mapuche. En: CONTRERAS PAINEMAL, C. Actas del primer congreso internacional de historia Mapuche. Uppsala: Ñuke Mapuförlaget, 2002, p. 27-45.

MARIMÁN, J. El conflicto nacionalitario y sus perspectivas de desarrollo en Chile. El caso Mapuche. Austerra. Revista de Antropología Social, 2005, № 2, p. 2-14.

MARIMÁN, P.; CANIUQUEO, S.; MILLALÉN, J. y LEVIL, R. i...Escucha, winka...! Cuatro ensayos de historia nacional Mapuche y un epílogo sobre el futuro. Santiago de Chile: LOM Ediciones, 2006.

MARSTON, S.A.; JONES, J.P. \& WOODWARD, K. Human geography without scale. Transactions of the Institute of British Geographers, 2005, Vol. 30, N4, p. 416-432.

MIGNOLO, W. La retórica del desarrollo y la colonialidad del saber. Página 12, 2009. Disponible en Internet:

http://www.pagina12.com.ar/diario/universidad/10-124958-2009-05-15.html

MOLINA, R. y CORREA, M. Territorios y comunidades Pehuenches del Alto Bio-Bio. Santiago de Chile: Corporación Nacional de Desarrollo Indígena, CONADI, 1998.

MOLINA, R.; CORREA, M. y BARCHIESI, C. Plan de desarrollo Pehuenche para el Area de Desarrollo Indígena del Alto Bio Bio. Santiago de Chile: informe preparado por el Grupo de Investigación TEPU (Territorios y Pueblos Indígenas) por mandato de la Corporación Nacional de Desarrollo Indígena, CONADI, 1998.

MORALES, H. Turismo comunitario. Una nueva alternativa de desarrollo indígena. En: MONETA, C. El jardín de los senderos que se encuentran. Políticas públicas y diversidad cultural en el MERCOSUR. Montevideo: UNESCO, 2006, p. 239-256.

MOWFORTH, M.; CHARLTON, C. \& MUNT, I. Tourism and responsibility: perspectives from Latin America and the Caribbean. London/New York: Routledge, 2008. 
ÑANCULEF, J. El Trafkintun en el marco de la cosmovisión mapuche. 2015. Disponible en Internet: http://www.mapuexpress. $\operatorname{org} / ? \mathrm{p}=1930$

ORGANIZACIONES TERRITORIALES MAPUCHE. Propuesta de Organizaciones Territoriales Mapuche al Estado de Chile. Wallmapu, 2009.

PALOMINO-SCHALSCHA, M. Indigeneity, autonomy and new cultural spaces: The decolonisation of practices, being and place through tourism in Alto Biobío, Chile. Christchurch: Tesis doctoral, University of Canterbury, 2012.

PILQUIMÁN, M. y SKEWES, J.C. Los paisajes locales y las encrucijadas del etnoturismo. Reflexiones a partir de los proyectos turísticos de comunidades indígenas de la región de Los Lagos en Chile. Cuadernos de Turismo, 2009, No 24, p. 169-191.

PINTO, J. La formación del Estado y la nación, y el pueblo Mapuche. De la inclusión a la exclusión. Santiago de Chile: Ediciones de la Dirección de Bibliotecas, Archivos y Museos DIBAM, 2003.

POLANYI, K. The livelihood of man. New York: Academic Press, 1977.

POLLARD, J.; MCEWAN, C. \& HUGHES, A. Postcolonial economies. London/New York: Zed Books, 2011.

PRADA, R. Más allá del capitalismo y la modernidad. En: GONSÁLVEZ, G. Descolonización en Bolivia. Cuatro ejes para comprender el cambio. Descolonización, estado plurinacional, economía plural y socialismo comunitario. La Paz: Vicepresidencia del Estado-Presidencia de la Asamblea Legislativa Plurinacional, 2010.

QUIDEL, J.; DURÁN, T. y CATRIQUIR, D. Rukache o familia mapunche: Una relación entre reñma y mapu. En: DURÁN, T.; CATRIQUIR, D. y HERNÁNDEZ, A. Patrimonio cultural mapunche: Derechos sociales y patrimonio institucional mapunche. Volumen III. Temuco: Editorial UC Temuco, 2007.
QUIJANO, A. Coloniality and modernity/ relationality. Cultural Studies, 2007, Vol 21, $\mathrm{N}^{\circ} 2$, p. 168-178.

QUIJANO, A. "Solidaridad" y capitalismo colonial/moderno. América Latina en Movimiento, 2008, Vol. 430, №32, p. 4-8.

ROELVINK, G. \& GIBSON-GRAHAM, J. K. A postcapitalist politics of dwelling: Ecological humanities and community economies in conversation. Australian Humanities Review, 2009, No 46, p. 145-158.

RUIZ-BALLESTEROS, E. \& HERNÁNDEZRAMíREZ, M. Tourism that empowers? Commodification and appropiation in Ecuador's Turismo Comunitario. Critique of Antrhopology, 2010, Vol 30, № 2, p. 201-229.

RUPAILAF MAICHIN, R. Reseña de "La mirada comunitaria, vínculos entre la ciencia occidental post-moderna y las sabidurías ancestrales aymara y mapuche" de Patricia Yungue. Polis, 2006, Vol. 5, № 15, p. 256-258.

SANTOS, B. Crítica de la razón indolente: Contra el desperdicio de la experiencia. Para un nuevo sentido común: la ciencia, el derecho y la política en la transición paradigmática. Bilbao: Editorial Desclée de Brouwer, 2003.

SPILLER, C.; ERAKOVIC, L.; HENARE, M. \& PIO, E. Relational well-being and wealth: Māori business and an ethic of care. Journal of Business Ethics, 2011, Vol. 98, № 1, p. 153-169.

TRICOT, T. El nuevo movimiento mapuche: Hacia la (re)construcción del mundo y el país Mapuche. Polis, 2009, Vol. 8, № 24, p. 175-196.

WEBER, M. Economy and society. An outline of interpretive sociology. Berkeley: University of California Press, 1968.

ZEIN-ELABDIN, E. \& CHARUSHEELA, S. Postcolonialism meets economics. London: Routledge, 2004. 
